\title{
Four point parabolic interpolation *
}

\author{
Michael A. Lachance \\ Department of Mathematics, University of Michigan-Dearborn, Dearborn, MI 48128, USA \\ Arthur J. Schwartz \\ Depariment of Mathematics, University of Michigan, Ann Arbor, MI 48109, USA
}

Received December 1989

Revised August 1990

Abstract

Lachance. M.A. and A.J. Schwartz. Four point parabolic interpolation, Computer Aided Geometric Design 8 (1991) 143-149.

We show that four points in the plane may be interpolated by one or two parabolas or possibly by no parabola, depending on the configuration of points. We provide methods for distinguishing the cases and constructing the parabolas.

\section{Introduction}

Polynomial and spline interpolation of digitized data are widely used in computer-aided design. In a number of systems, most notably Ford Motor Company's Product Design Graphics System (PDGS), parabolic interpolation is employed. As implemented in PDGS, three points $P_{0}, P_{1}$ and $P_{2}$ are uniquely interpolated by the parametric parabola, $P(t)=A t^{2}+$ $B t+C$, satisfying

$$
P(0)=P_{0}, \quad P(0.5)=P_{1}, \quad P(1)=P_{2} .
$$

This is the so-called Cinci parabola. the name being coined by the US tool manufacturer Cincinnati Millicron.

The Cinci parabola is not the only parabola interpolating three given noncollinear points. In fact, an infinite number of distinct parabolas, $P_{\alpha}(t)$, where $\alpha \in \mathbb{R}-\{0,1\}$, and which satisfy

$$
P_{\alpha}(0)=P_{0}, \quad P_{\alpha}(\alpha)=P_{1}, \quad P_{\alpha}(1)=P_{2}
$$

will do so.

It is natural to ask if the specification of a fourth (coplanar) point, $P_{3}$, will determine a parabola. By sketching one parabola superimposed upon another, one can see that it is possible for two parabolas to contain the same four points. It is also intuitively true that no parabola interpolates the vertices of a square. In this paper we show that four given points may belong to two, one or no parabolas as follows: if the four points are the vertices of a convex quadrilateral which is not a trapezoid, then there are exactly two parabolas containing those points; if the points are the vertices of a trapezoid but not a parallelogram, then there is exactly one interpolating parabola; otherwise, no parabola interpolates the points. We provide a very simple method for finding parametrizations and defining equations for the parabolas in

\footnotetext{
* Research for this paper was partially supported by the Ford Motor Company.
} 
question. We obtain our results using standard tools of linear algebra and affine geometry and by developing a connection between the parametric and implicit forms of parabolas. .

For the sake of brevity we have left out a number of fairly straightforward computations and proofs. We have also left out a number of related issues of affine geometry which are suggested by the problem at hand. This discussion, along with a great deal more detail, may be found in [Lachance \& Schwartz].

We are grateful to the referee who pointed out that the problem of constructing a parabola through four given points was addressed by Newton [Dörrie]. That construction uses a Euclidean approach and is fairly difficult. It does not directly describe the relationship between the configuration of the points and the number of interpolating parabolas.

\section{Definitions and notation}

Definition 1. If for a nonsingular real $2 \times 2$ matrix $A^{\prime}$ and for constants $d, e \in \mathbb{R}$,

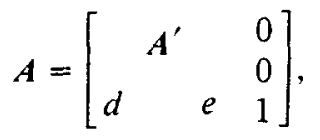

the matrix $\boldsymbol{A}$ is called an affine matrix. The apparently superfluous column, $(0,0,1)^{\mathrm{T}}$, guarantees that the set of affine matrices will satisfy a number of useful properties; for example, it is closed under matrix multiplication and inverse operations.

We use affine matrices to establish an equivalence relation on the set of real symmetric $3 \times 3$ matrices. We say that two matrices $B$ and $C$ are affinely congruent if there exists an affine matrix $\boldsymbol{A}$ for which $B=A C A^{\mathrm{T}}$. We will denote this congruence by $B \approx C$.

Definition 2. We denote the matrix

$$
D:=\left[\begin{array}{rrr}
0 & 0 & 1 \\
0 & -2 & 0 \\
1 & 0 & 0
\end{array}\right] \text {. }
$$

We specify a particular set of $3 \times 3$ symmetric matrices

$$
\mathscr{P}:=\{\boldsymbol{B}: \boldsymbol{B} \approx d \boldsymbol{D} \text {, for some } d \neq 0\} .
$$

A parabola is a plane set of the form

$$
C_{B}=\left\{(x, y) \mid(x, y, 1) B(x, y, 1)^{\mathrm{T}}=0\right\} .
$$

When $B=D, C_{B}$ corresponds to the familiar $x=y^{2}$. In other cases, $C_{B}$ is an affine image of $C_{D}$.

\section{Parametrized and implicit parabolas}

We now develop a connection between the parametrized forms of parabolas and their implicit forms.

Definition 3. Let $p(t), q(t)$, and $r(t) \equiv 1$ be linearly independent polynomials of degree at most two. If for some $B \in \mathscr{P}$,

$$
C_{B}=\{(p(t), q(t)) \mid t \in \mathbb{R}\},
$$

we say that $(p(t), q(t), 1)$ is a quadratic parametrization of $C_{B}$. 
Proposition 1. Let $p(t), q(t)$, and $r(t) \equiv 1$ be three linearly independent polynomials of degree at most two. let the coefficient matrix $C$ be defined so that $(p(t), q(t), 1)=\left(t^{2}, t, 1\right) C$, and let $\boldsymbol{B} \in \mathscr{P}$. Then

(i) $C$ is an affine matrix, and

(ii) $(p(t), q(t), 1)$ is a quadratic parametrization of $C_{B}$ if and only if $C B C^{\top}=d D$, for some $d \neq 0$.

Proof. Since $p(t), q(t)$ and 1 are linearly independent $C$ must be nonsingular. Since the last component of $\left(t^{2}, t, 1\right) C$ is 1 it follows that the last column of $C$ is $(0,0.1)^{\mathrm{T}}$ and therefore that $C$ is affine. The remainder of the proposition follows from the fact that

$$
\begin{aligned}
0 & =(p(t), q(t), 1) B(p(t), q(t), 1)^{\mathrm{T}} \\
& =\left(t^{2}, t, 1\right) C B C^{\mathrm{T}}\left(t^{2}, t, 1\right)^{\mathrm{T}} \\
& =\left(t^{2}, t, 1\right)\left[\begin{array}{lll}
a & b & d \\
b & c & e \\
d & e & f
\end{array}\right]\left[\begin{array}{c}
t^{2} \\
t \\
1
\end{array}\right] \\
& =a t^{4}+2 b t^{3}+(c+2 d) t^{2}+2 e t+f
\end{aligned}
$$

holds identically if and only if $a=b=e=f=0$ and $c+2 d=0$.

Corollary 1. Every parabola has a quadratic parametrization.

Corollary 2. Let $\boldsymbol{B}$ and $\boldsymbol{B}^{*} \in \mathscr{P}$. Then $C_{\boldsymbol{B}}=C_{\boldsymbol{B}}$. if and only if $\boldsymbol{B}=d \boldsymbol{B}^{*}$ for some $d \neq 0$.

\section{Parabolic interpolation}

We first consider the problem of interpolating a given triple of points, $Q_{1}, Q_{2}, Q_{3}$ in $\mathbb{R}^{2}$.

Definition 4. For $\alpha \in \mathbb{R}$, we define the Vandermonde Matrix,

$$
V(\alpha):=\left[\begin{array}{ccc}
0 & 0 & 1 \\
\alpha^{2} & \alpha & 1 \\
1 & 1 & 1
\end{array}\right]
$$

For $Q_{1}, Q_{2}$, and $Q_{3}$ we define the configuration matrix

$$
R\left(Q_{1}, Q_{2}, Q_{3}\right):=\left[\begin{array}{ll}
Q_{1} & 1 \\
Q_{2} & 1 \\
Q_{3} & 1
\end{array}\right] .
$$

Proposition 2. Let $Q_{1}, Q_{2}, Q_{3}$ be distinct collinear points. Then no parabola contains them.

Proof. Every parabola can be parametrized by $\left(t^{2}, t, 1\right) C$ for some affine matrix $C$. Any three distinct points will correspond to three distinct values of $t$, to three linearly independent values of $\left(t^{2}, t, 1\right)$ and, since $C$ is affine, to three noncollinear points.

Proposition 3. Let $Q_{1}, Q_{2}, Q_{3}$ be noncollinear points. The unique quadratic parametrization, $(p(t), q(t), 1)$, which satisfies $(p(0), q(0))=Q_{1},(p(\alpha), q(\alpha))=Q_{2}$ and $(p(1), q(1))=Q_{3}$ is given $b y$

$$
\Phi_{\alpha}\left(t ; Q_{1}, Q_{2}, Q_{3}\right):=\left(t^{2}, t, 1\right) V(\alpha)^{-1} \boldsymbol{R}\left(Q_{1}, Q_{2}, Q_{3}\right)
$$


Definition 5. Let $Q_{1}, Q_{2}$ and $Q_{3}$ be noncollinear points in $\mathbb{R}^{2}$. We define $\mathfrak{P}\left(Q_{1}, Q_{2}, Q_{3}\right)$ to be the set of parabolas containing these points.

Convention. When $Q_{1}, Q_{2}$, and $Q_{3}$ are determined by the context, we abbreviate:

$$
\begin{aligned}
& R=R\left(Q_{1}, Q_{2}, Q_{3}\right), \\
& \Phi_{\alpha}(t)=\Phi_{\alpha}\left(t ; Q_{1}, Q_{2}, Q_{3}\right), \\
& \mathfrak{P}=\mathfrak{B}\left(Q_{1}, Q_{2}, Q_{3}\right) .
\end{aligned}
$$

We also need the following definitions:

\section{Definition 6.}

$$
A_{\alpha}:=V(\alpha) D(V(\alpha))^{\top}
$$

and

$$
B_{\alpha}:=R^{-1} A_{\alpha}\left(R^{-1}\right)^{\mathrm{T}}
$$

We are now able to state

Proposition 4. Given noncollinear points $Q_{1}, Q_{2}$ and $Q_{3}$, the map $\alpha \mapsto B_{\alpha}$ defines a correspondence which is $1-1$ and onto between $\mathbb{R}-\{0,1\}$ and $\mathfrak{B}$.

Proof. According to Proposition 3, for each $\alpha \neq 0,1, \Phi_{\alpha}(t)$ parametrizes a parabola, $C_{B} \in \mathfrak{B}$. Conversely, according to Corollary 1 of Proposition 1 , if $C_{\boldsymbol{B}} \in \mathfrak{R}$ it may be quadratically parametrized by some $\Psi(t)$. If $\Psi\left(t_{i}\right)=\left(Q_{i}, 1\right)$, we set $\alpha=\left(t_{2}-t_{1}\right) /\left(t_{3}-t_{1}\right)$ and note that $\Phi_{\alpha}(t)=\Psi\left(t_{1}+\left(t_{3}-t_{1}\right) t\right)$ also parametrizes $C_{B}$. Thus $C_{B} \in \mathfrak{B}$ if and only if it is parametrized by $\Phi_{\alpha}(t)$, for some $\alpha \neq 0,1$.

According to Proposition 1 and its second corollary, it is parametrized by $\Phi_{\alpha}(t)$ if and only if $B=d B_{\alpha}$ for some $d \neq 0$. The result now follows from Proposition 3, its corollaries and the observation that $\alpha \neq \beta$ implies that $A_{\alpha} \neq d A_{\beta}$ for any $d$.

Given three noncollinear points, $Q_{1}, Q_{2}$ and $Q_{3}$ we may introduce an oblique coordinate system with respect to these points. That is to say, given an arbitrary point, $Q$, we can find $q=\left(q_{1}, q_{2}, q_{3}\right)$ such that $(Q, 1)=\sum_{i=1}^{3} q_{i}\left(Q_{i}, 1\right)=q R$. We see that $q=(Q, 1) R^{-1}$.

Proposition 5. For $q$ defined above $q_{1}+q_{2}+q_{3}=1$ and $q_{i}=0$ if and only if $Q$ is collinear with $Q_{j}$ and $Q_{k}$, the points distinct from $Q_{i}$.

We turn our attention to the four point interpolation problem. We will denote by $Q=$ $\left\{Q_{1}, Q_{2}, Q_{3}, Q_{4}\right\}$ an arbitrary quadrupole of points no three of which are collinear. We shall say that such a quadruple of points forms a convex quadrilateral if no $Q_{i}$ is in the convex hull of the remaining three.

Proposition 6. The quadruple $Q=\left\{Q_{1}, Q_{2}, Q_{3}, Q_{4}\right\}$ forms a convex quadrilateral if and only if $q_{1} q_{2} q_{3}<0$, where $\left(Q_{4}, 1\right)=q \boldsymbol{R}\left(Q_{1}, Q_{2}, Q_{3}\right)$. (Sce Fig. 1.)

Theorem 1. Let $Q=\left\{Q_{1}, Q_{2}, Q_{3}, Q_{4}\right\}$ be a quadruple of points no three of which are collinear. Then

(i) If $Q$ does not form a convex quadrilateral, then there is no parabola interpolating $Q$.

(ii) If $Q$ is the vertex set of a parallelogram, then there is no parabola interpolating $Q$. 


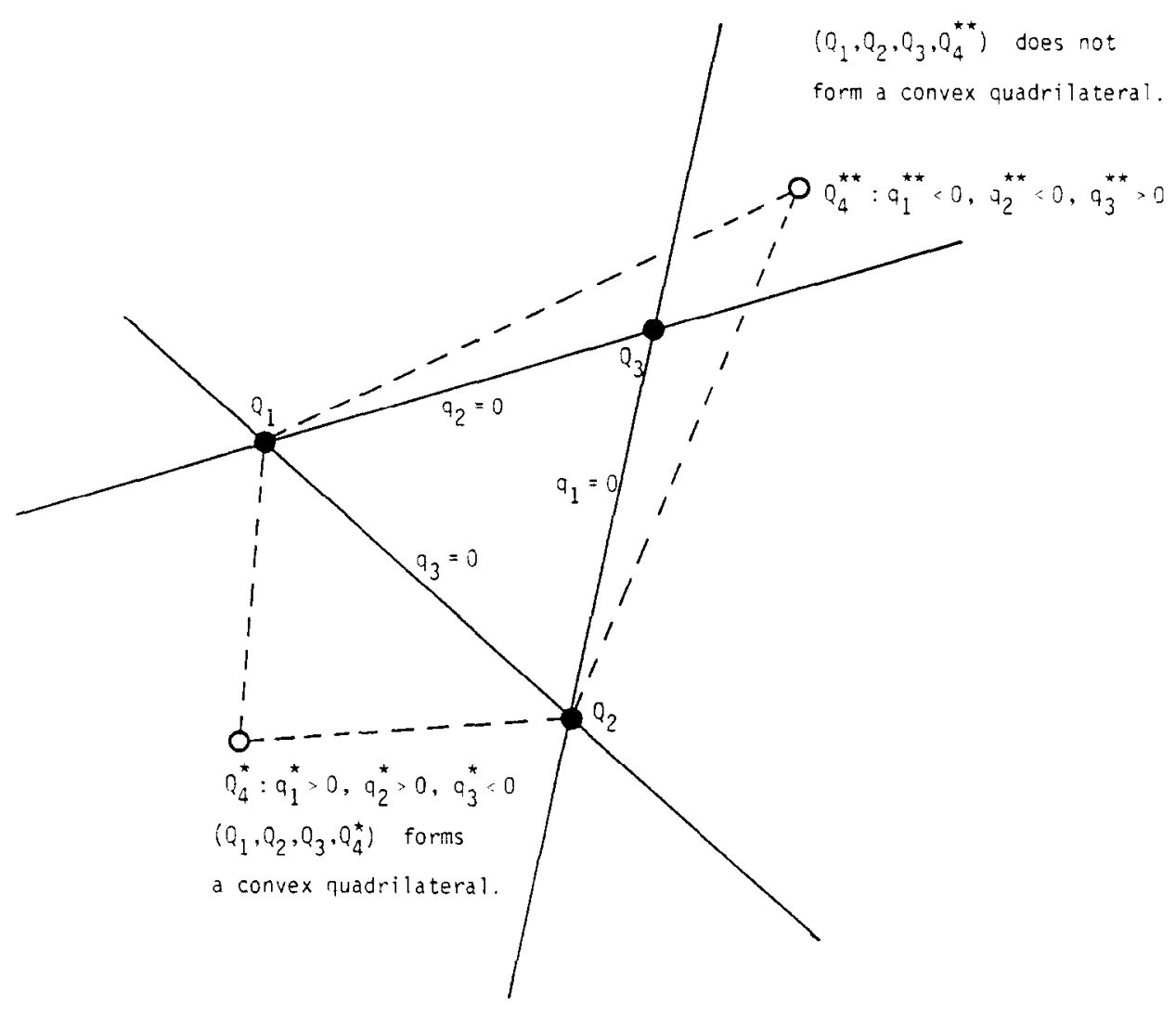

Fig. 1.

(iii) If $Q$ is the vertex set of a trapezoid and not a parallelogram, then there is exactly one parabola interpolating $Q$.

(iv) If $Q$ forms a convex quadrilateral and not a trapezoid, then there are exactly two parabolas interpolating $Q$.

Proof. We defined $\Phi_{\alpha}(t), \boldsymbol{A}_{\alpha}$ and $\boldsymbol{B}_{\alpha}$ as in (10), (12) and (13). According to Proposition 4, $Q_{4}$ is contained in a parabola of $\mathfrak{W}$ for each $\alpha \neq 0,1$, such that $Q_{4}$ is on the parabola $C_{\boldsymbol{B}_{\alpha}}$. i.e., when

$$
\begin{aligned}
0 & =\left(Q_{4}, 1\right) \boldsymbol{B}_{\alpha}\left(Q_{4}, 1\right)^{\mathrm{T}} \\
& =\left(Q_{4}, 1\right) \boldsymbol{R}^{-1} \boldsymbol{A}_{\alpha}\left(\boldsymbol{R}^{-1}\right)^{\mathrm{T}}\left(Q_{4}, 1\right)^{\mathrm{\top}} \\
& =\left(q_{1}, q_{2}, q_{3}\right) \boldsymbol{A}_{\alpha}\left(q_{1}, q_{2}, q_{3}\right)^{\mathrm{T}} \\
& =\alpha^{2} q_{1} q_{2}+q_{1} q_{3}+(\alpha-1)^{2} q_{2} q_{3}
\end{aligned}
$$

or

$$
0=\alpha^{2} q_{2}\left(1-q_{2}\right)-2 \alpha q_{2} q_{3}+q_{3}\left(1-q_{3}\right) .
$$

We remind the reader that $\sum_{1}^{3} q_{i}=1, q_{1} q_{2} q_{3} \neq 0$ and consider the following facts:

(a) The discriminant of (15) is $-4 q_{1} q_{2} q_{3}$ (which may be obtained after a moderate amount of calculation) so that there are no double roots. 


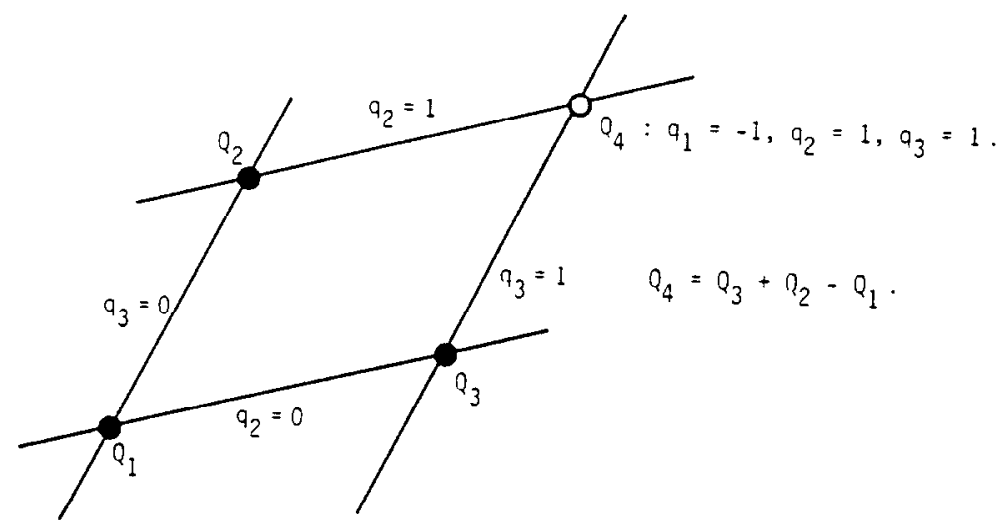

Fig. 2.

(b) (15) has a unique solution for $\alpha$ if and only if $q_{2}=1$.

(c) (15) has $\alpha=0$ as one of its solutions if and only if $q_{3}=1$.

(d) (14) has $\alpha=1$ as one of its solutions if and only if $q_{1}=1$.

From (b), (c) and (d) we see that if $q_{i}=1$ for two values of $i$, then there is no value of $\alpha$ satisfying (15) other than 0 or 1 and thus no parabola interpolating $Q$. In this case $Q$ forms a parallelogram (see Fig. 2).

Considering (b), (c) and (d) also reveals that if $q_{i}=1$ for exactly one $i$, then there is one value of $\alpha$ other than 0 or 1 satisfying (15), hence according to Proposition 4, one parabola interpolating $Q$. In this case $Q$ forms a trapezoid but not a parallelogram (see Fig. 3).

If $q_{i}=1$ for no value of $i$, then $Q$ does not form a trapezoid. According to Proposition 6 it forms a convex quadrilateral if and only if $-4 q_{1} q_{2} q_{3}>0$ which is the case if and only if (14) is satisfied by two values of $\alpha$ and thus $Q$ is interpolated by two parabolas. Finally, if $-4 q_{1} q_{2} q_{3}<0$, there is no parabola interpolating $Q$ which in turn does not form a convex quadrilateral.

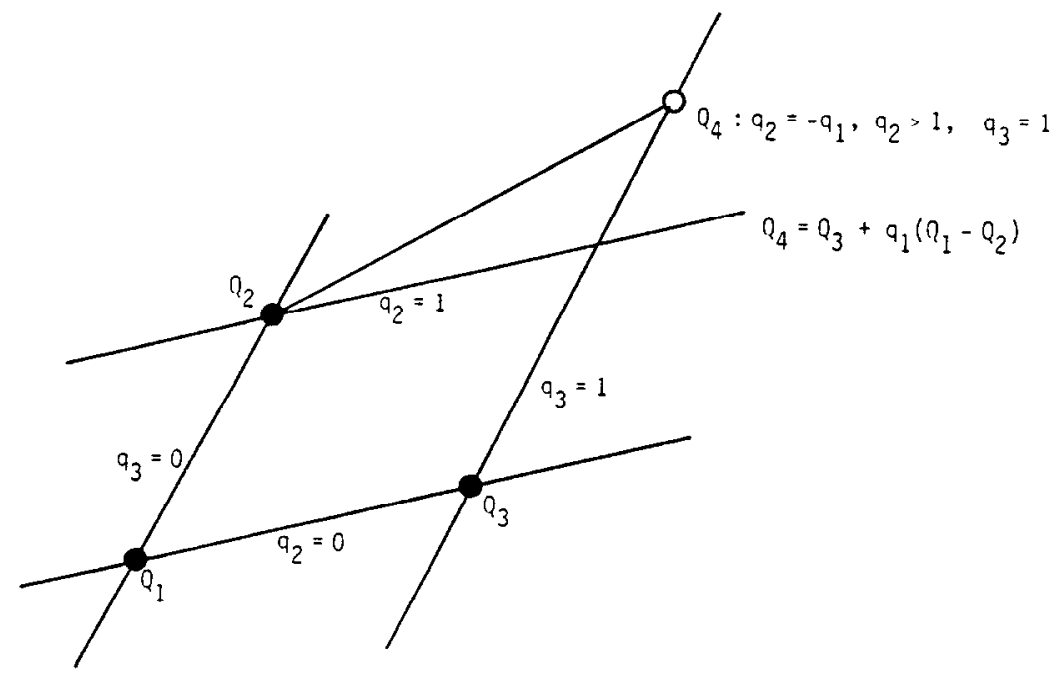

Fig. 3. 
Finally, we note that to find the parabolas, if they exist, we merely need to compute $q_{1}, q_{2}$ and $q_{3}$ and solve (14) for $\alpha$. The parametrization is given by $\Phi_{\alpha}(t)$ and the defining matrix is given by $\boldsymbol{B}_{\alpha}$.

\section{References}

Dörrie, H., 100 Great Problems of Elementary Mathematics, 2nd edition, Dover, New York.

Lachance, M. and A. Schwartz, Matrix factorization methods in quadratic interpolation, University of Michigan Reprint. 